\title{
Experimental Study of Indoor Tracking Using UWB Measurements and Particle Filtering
}

\author{
Vladimir Savic and Erik G. Larsson
}

\section{Linköping University Post Print}

\section{Tweet}

N.B.: When citing this work, cite the original article.

Original Publication:

Vladimir Savic and Erik G. Larsson, Experimental Study of Indoor Tracking Using UWB Measurements and Particle Filtering, 2016, 17th IEEE International Workshop on Signal Processing Advances in Wireless Communications (SPAWC 2016), 3-6 July 2016, Edinburgh, UK.

Copyright: The Authors.

Preprint ahead of publication available at: Linköping University Electronic Press http://urn.kb.se/resolve?urn=urn:nbn:se:liu:diva-128263 


\title{
Experimental Study of Indoor Tracking Using UWB Measurements and Particle Filtering
}

\author{
Vladimir Savic and Erik G. Larsson \\ Dept. of Electrical Engineering (ISY), Linköping University, Linköping, Sweden \\ Emails: vladimir.savic@liu.se, erik.larsson@isy.liu.se
}

\begin{abstract}
Target tracking with ultra-wideband (UWB) signals in indoor environments is a challenging problem due to the presence of multipath and non-line-of-sight conditions (NLOS). A solution to this problem is to use particle filtering (PF), which is able to handle both nonlinear models and non-Gaussian uncertainties that typically appear in the presence of NLOS. In this paper, we compare four different PF variants, that differ in terms of how NLOS measurements are handled. According to our experimental results, based on the measurements from a basement tunnel, multiple features from the UWB impulse response should be used, and the ranging likelihood function should make use of both LOS and NLOS measurements. Standard timeof-arrival (TOA) based methods, even with NLOS rejection, are not good enough. Instead we advocate TOA-based algorithms that can actively mitigate errors due to NLOS.
\end{abstract}

Index Terms - target tracking, ultra-wideband, particle filtering, machine learning, time of arrival.

\section{INTRODUCTION}

Target tracking in indoor environments [1], [2] is an important problem for many applications, including search-andrescue operations, production monitoring, and traffic management. However, we cannot rely on Global Positioning System (GPS) due to its unavailability in indoor environments. Alternative technologies may be based on RFID, WiFi, Bluetooth and UWB. The most promising one is based on UWB radios [3] since they can be easily deployed indoors, have large communication range, and can provide very high ranging precision. Nevertheless, it also has a problem with NLOS measurements, when the range estimates are positively biased. This problem may be solved [4], [5] either by rejecting the samples, or mitigating the error using the available information from the UWB signal. Indoor tracking with UWB technology has been mainly done within a Bayesian framework. The main methods are based on variants of Kalman filtering (KF) and particle filtering (PF). Although $\mathrm{KF}$ methods have low complexity, PF methods are able to handle both nonlinear relationships and non-Gaussian uncertainties that typically appear in the presence of NLOS. Regarding the measurements, most of the methods are based on TOA since UWB signals have very high time-resolution. However, it is preferable to use additional signal features especially for NLOS identification and error mitigation. Regarding the dynamic model, we can either use different types of the random walk models, or make use of IMUs to measure the acceleration. A detailed review 978-1-5090-1749-2/16/\$31.00 (C)2016 IEEE of indoor tracking methods is available in [1].

In this paper, we compare four different PF variants, that differ in terms of how NLOS measurements are handled. More specifically, we use four different models for the likelihood function: i) direct TOA-based ranging, ii) TOA-based ranging with the rejection of NLOS measurements, iii) TOA-based ranging with mitigation of NLOS bias, and iv) TOA-based ranging with Gaussian Process Regression (GPR) based estimation for the NLOS case. We compare these methods using UWB measurements from a basement tunnel at Linköping university [6]. According to our results, the latter two methods provide the best performance since they make use of multiple features from the UWB impulse response from both LOS and NLOS measurements. The other two options are not good choices due to the accumulation of error over time.

The remainder of this paper is organized as follows. In Section II, we describe the system model and the tracking algorithm based on PF. Then, in Section III, we define four different likelihood models for range estimation. The experimental results, based on UWB measurements from a basement tunnel, are provided in Section IV. Finally, in Section V, we summarize our conclusions and discuss future directions.

\section{The tracking Algorithm}

We assume that there are $N_{b}$ static beacons with known 2-dimensional (2D) positions and one mobile target with an unknown position. Both the beacons and the target are equipped with UWB radios, and the target is also equipped with an IMU. The goal is to track the target using available UWB measurements from all beacons. The tracking algorithm is executed either by a central unit or the target itself. ${ }^{1}$ For this problem, we use the following state-space model:

$$
\begin{aligned}
& \mathbf{x}_{t+1}=\mathbf{x}_{t}+T \mathbf{u}_{t}+\mathbf{w}_{t} \\
& y_{n, t}=\left\|\mathbf{l}_{n}-\mathbf{x}_{t}\right\|+v_{n, t}
\end{aligned}
$$

where $\mathbf{x}_{t}=\left[x_{1, t} x_{2, t}\right]^{T}$ is an unknown 2D position of the target at time $t\left(t=1, \ldots, N_{t}\right) ; \mathbf{u}_{t}=\left[\begin{array}{ll}u_{1, t} & u_{2, t}\end{array}\right]^{T}$ is the velocity at time $t$ obtained by integrating the acceleration measured by the IMU; $\mathbf{w}_{t}=\left[\begin{array}{ll}w_{1, t} & w_{2, t}\end{array}\right]^{T}$ is the process noise $\left(\mathbf{w}_{t} \sim p_{w}(\cdot)\right)$; $T$ is the sampling interval; $y_{n, t}$ is the measured distance between beacon $n$ and the target at time $t ; \mathbf{l}_{n}=\left[\begin{array}{ll}l_{1, n} & l_{2, n}\end{array}\right]^{T}$

\footnotetext{
${ }^{1}$ Alternatively, the beacons may agree on the target state via distributed algorithms [7], but this problem is out of scope of this paper.
} 
is the known position of beacon $n\left(n=1, \ldots, N_{b}\right)$; and $v_{n, t}$ is the measurement noise $\left(v_{n, t} \sim p_{v}(\cdot)\right)$.

We apply Bayesian filtering for this problem, so our goal is to recursively determine the posterior $p\left(\mathbf{x}_{t} \mid \mathbf{y}_{1: t}\right)$, given the prior $p\left(\mathbf{x}_{t-1} \mid \mathbf{y}_{1: t-1}\right)$, the prediction $p\left(\mathbf{x}_{t} \mid \mathbf{x}_{t-1}\right)$, and the likelihood $p\left(\mathbf{y}_{t} \mid \mathbf{x}_{t}\right)$. We assume that $p\left(\mathbf{x}_{0} \mid \mathbf{y}_{0}\right)=p\left(\mathbf{x}_{0}\right)$ is available a priori (if not available, the prior is initialized with uniform distribution over whole area). Therefore, the posterior can be found as:

$$
p\left(\mathbf{x}_{t} \mid \mathbf{y}_{1: t}\right) \propto p\left(\mathbf{y}_{t} \mid \mathbf{x}_{t}\right) \int p\left(\mathbf{x}_{t} \mid \mathbf{x}_{t-1}\right) p\left(\mathbf{x}_{t-1} \mid \mathbf{y}_{1: t-1}\right) \mathrm{d} \mathbf{x}_{t-1}
$$

Taking the models (1)-(2) into account, the prediction and likelihood are given by:

$$
\begin{gathered}
p\left(\mathbf{x}_{t} \mid \mathbf{x}_{t-1}\right)=p_{w}\left(\mathbf{x}_{t}-\mathbf{x}_{t-1}-T \mathbf{u}_{t-1}\right) \\
p\left(\mathbf{y}_{t} \mid \mathbf{x}_{t}\right)=\prod_{n=1}^{N_{b}} p\left(y_{n, t} \mid \mathbf{x}_{t}\right)=\prod_{n=1}^{N_{b}} p_{v}\left(y_{n, t}-\left\|\mathbf{l}_{n}-\mathbf{x}_{t}\right\|\right)
\end{gathered}
$$

While for $p_{w}(\cdot)$ it is reasonable to assume a $2 \mathrm{D}$ zero-mean Gaussian distribution (with covariance matrix $\Sigma_{w}$ ), the model for $p_{v}(\cdot)$ is expected to be more complex due to the presence of NLOS conditions. This problem will be considered in the next section.

Since the range measurement is not a linear function of the state (see (2)), and the measurement noise is not Gaussian in some cases (see Section III), a traditional Kalman filtering approach [8] is not recommended. Therefore, we use the particle filtering (PF) [9] in which the posterior is represented by a set of samples (also called, particles) with associated weights. More specifically, we apply the standard sampleimportance-resampling (SIR) PF algorithm in which we draw $N_{p}$ particles from $p\left(\mathbf{x}_{t} \mid \mathbf{x}_{t-1}\right)$, then compute the weights using the likelihood $p\left(\mathbf{y}_{t} \mid \mathbf{x}_{t}\right)$, and finally resample with replacement to avoid the degeneracy problem (i.e., when all but very few particle have negligible weights). Assuming that we have a set of particles $\left\{w_{t-1}^{(m)}, \mathbf{x}_{t-1}^{(m)}\right\}\left(m=1, \ldots, N_{p}\right)$ representing $p\left(\mathbf{x}_{t-1} \mid \mathbf{y}_{1: t-1}\right)$ (initially, $\left\{w_{0}^{(m)}, \mathbf{x}_{0}^{(m)}\right\} \sim p\left(\mathbf{x}_{0}\right)$ ), the PF-SIR algorithm (at time $t$ ) proceeds as follows:

1) Draw $N_{p}$ particles: $\mathbf{x}_{t}^{(m)} \sim p\left(\mathbf{x}_{t} \mid \mathbf{x}_{t-1}^{(m)}\right)$

2) Compute the weights: $w_{t}^{(m)}=w_{t-1}^{(m)} \cdot p\left(\mathbf{y}_{t} \mid \mathbf{x}_{t}^{(m)}\right)$

3) Normalize: $w_{t}^{(m)}=w_{t}^{(m)} / \sum_{m^{\prime}=1}^{N_{p}} w_{t}^{\left(m^{\prime}\right)}$

4) Compute the estimates: $\hat{\mathbf{x}}_{t}=\sum_{m=1}^{N_{p}} w_{t}^{(m)} \mathbf{x}_{t}^{(m)}$

5) Resample with replacement from $\left\{w_{t}^{(m)}, \mathbf{x}_{t}^{(m)}\right\}_{m=1}^{N_{p}}$

\section{LIKELIHOOD MODELS}

Our goal is to estimate the distance using the UWB signal received from the beacon. We decided to use a measured complex impulse response $(\mathrm{CIR})^{2}$ of the channel, given by $h(t)=\sum_{k} a_{k} \delta\left(t-\tau_{k}\right)$ where $a_{k} \in \mathbb{C}, k=1, \ldots, N_{k}$, and $\tau_{k}$ is the delay of the $k$-th path. We can then extract a number

\footnotetext{
${ }^{2}$ Alternatively, the output waveforms may be used in a similar way.
}

of features from the CIR, that provide us some information about the unknown range and about whether the measurements was taken in LOS or NLOS. We found that time-of-arrival (TOA) is the most useful for ranging (as expected), rise-time is the most useful for NLOS identification, and the maximumexcess-delay is most useful for NLOS bias mitigation. For the GPR-based machine learning method, we additionally use received signal strength, maximum receiver power, mean excess delay, RMS delay spread, and kurtosis (in total, 8 features). A justification for the use of these features and their definitions are available in [6].

Then, motivated by our previous research results [6], [10], we consider four options for the likelihood model:

1) Direct TOA-based ranging: We assume that the measurement, also known as pseudo-range, is simply given by $y_{n, t}=c \tau_{n, t}$ where $\tau_{n, t}$ is the time-of-arrival (TOA) between beacon $n$ and the target at time $t$, and $c \approx 3 \cdot 10^{8}$ $\mathrm{m} / \mathrm{s}$ is the speed of light. Then, the likelihood is given by:

$$
p\left(y_{n, t} \mid \mathbf{x}_{t}\right)=\mathcal{N}\left(c \tau_{n, t}-\left\|\mathbf{l}_{n}-\mathbf{x}_{t}\right\| ; \mu_{L}, \sigma_{L}^{2}\right)
$$

where $\mu_{L}$ and $\sigma_{L}^{2}$ represent, empirically estimated, mean and the variance of the LOS ranging error. Note that here we do not have any information about the NLOS error statistics, so this approach will typically lead to positively biased range estimates.

2) TOA-based ranging with rejection of NLOS measurements $(T O A+R e j e c t)$ : In this case, we would like to use only LOS measurements, so we need to detect whether the measurement is taken in NLOS conditions. For that purpose, we define the binary variable $H_{n, t} \in$ $\{$ LOS, NLOS $\}$ and use likelihood $p\left(\tau_{n, t}^{R} \mid H_{n, t}\right)$ (where $\tau_{n, t}^{R}$ is the rise-time) for NLOS identification. According to our previous results [6], this likelihood follows an exponential distribution:

$$
p\left(\tau_{n, t}^{R} \mid H_{n, t}\right)= \begin{cases}\lambda_{L} e^{-\lambda_{L} \tau_{n, t}^{R},} & \text { if } H_{n, t}=\mathrm{LOS} \\ \lambda_{N} e^{-\lambda_{N} \tau_{n, t}^{R}}, & \text { if } H_{n, t}=\mathrm{NLOS}\end{cases}
$$

where the parameters $\lambda_{L}$ and $\lambda_{N}$ represent the decay rates, and are estimated from LOS and NLOS samples, respectively. Then, the probability of LOS condition is given by:

$$
\xi_{n, t}=\frac{p\left(\tau_{n, t}^{R} \mid H_{n, t}=\mathrm{LOS}\right)}{p\left(\tau_{n, t}^{R} \mid H_{n, t}=\mathrm{LOS}\right)+p\left(\tau_{n, t}^{R} \mid H_{n, t}=\mathrm{NLOS}\right)}
$$

Assuming that we want to reject the measurements that likely are NLOS (i.e., $\xi_{n, t}<\xi_{T H}$, where $\xi_{T H}$ is a predefined threshold, typically equal to $1 / 2$ ), the likelihood is given by:

$$
\begin{aligned}
& p\left(y_{n, t} \mid \mathbf{x}_{t}\right)= \\
& \begin{cases}\mathcal{N}\left(c \tau_{n, t}-\left\|\mathbf{l}_{n}-\mathbf{x}_{t}\right\| ; \mu_{L}, \sigma_{L}^{2}\right), & \text { if } \xi_{n, t}>\xi_{T H} \\
\text { const. } & \text { if } \xi_{n, t}<\xi_{T H}\end{cases}
\end{aligned}
$$


This approach may work if there are at least 3 LOS measurements, and if the rise-time can provide us certain information about the NLOS condition (i.e., if $\xi_{n, t}$ is not close to $1 / 2$ ). However, this may not be the case in many scenarios.

3) TOA-based ranging with mitigation of NLOS bias $(T O A+$ Mitig): Instead of rejecting the NLOS measurements, we make use of them with an appropriate model of the NLOS bias. The likelihood function which takes into account both hypotheses is given by [11]:

$$
\begin{gathered}
p\left(y_{n, t} \mid \mathbf{x}_{t}\right)=\xi_{n, t} \mathcal{N}\left(c \tau_{n, t}-\left\|\mathbf{l}_{n}-\mathbf{x}_{t}\right\| ; \mu_{L}, \sigma_{L}^{2}\right)+ \\
\left(1-\xi_{n, t}\right) \mathcal{N}\left(c \tau_{n, t}-\left\|\mathbf{l}_{n}-\mathbf{x}_{t}\right\| ; \mu_{N}, \sigma_{N}^{2}\right)
\end{gathered}
$$

The NLOS bias can be modeled as a nonlinear function of maximum excess delay, i.e., $\mu_{N}=g\left(\tau_{n, t}^{M}\right)$. Our results showed that the second-order polynomial model (with the coefficients $\left(p_{2}, p_{1}, p_{0}\right)$ estimated from NLOS data) provides a good approximation. The standard deviation of NLOS error $\sigma_{N}$ is also estimated from the same NLOS data set.

4) TOA-based ranging with GPR-based estimation for NLOS case $(T O A+G P R)$ : In this case, we keep using TOA for LOS case, but apply a nonparametric Bayesian machine learning method to estimate the range from NLOS samples. More specifically, we use Gaussian Process Regression (GPR) [12], which is able to model arbitrary nonlinear functions, given a measurement, and a set of training samples obtained in the considered environment. The likelihood is, as in the previous case, given in a mixture form:

$$
\begin{aligned}
& p\left(y_{n, t} \mid \mathbf{x}_{t}\right)=\xi_{n, t} \mathcal{N}\left(c \tau_{n, t}-\left\|\mathbf{l}_{n}-\mathbf{x}_{t}\right\| ; \mu_{L}, \sigma_{L}^{2}\right)+ \\
& \left(1-\xi_{n, t}\right) \mathcal{N}\left(\left\|\mathbf{1}_{n}-\mathbf{x}_{t}\right\| ; \mu_{G P R}(\mathbf{a}), \sigma_{G P R}(\mathbf{a})^{2}\right)
\end{aligned}
$$

where $\mu_{G P R}(\mathbf{a}), \sigma_{G P R}(\mathbf{a})$ represent the estimate of the mean and standard deviation of the distance ${ }^{3}$ and $\mathbf{a}$ is the normalized ${ }^{4}$ measurement vector (in this case, 8dimensional feature) obtained from UWB signal. They are given by:

$$
\begin{gathered}
\mu_{G P R}(\mathbf{a})=\mathbf{k}^{T}\left(\mathbf{K}+\sigma_{\omega}^{2} \mathbf{I}_{M}\right)^{-1} \mathbf{d}_{M} \\
\sigma_{G P R}(\mathbf{a})^{2}=\sigma_{d}^{2}+k(\mathbf{a}, \mathbf{a})-\mathbf{k}^{T}\left(\mathbf{K}+\sigma_{\omega}^{2} \mathbf{I}_{M}\right)^{-1} \mathbf{k}
\end{gathered}
$$

where $M$ is the number of training samples; $\mathbf{I}_{M}$ is the $M \times M$ identity matrix; $\sigma_{d}$ is the standard deviation of the measurement error; $\mathbf{d}_{M}=\left(d_{1}, \ldots, d_{M}\right)^{T}$ is a vector of true distances corresponding to training samples $\mathbf{a}_{1}, \ldots, \mathbf{a}_{M} ; \mathbf{k}=\left[k\left(\mathbf{a}_{1}, \mathbf{a}\right), k\left(\mathbf{a}_{2}, \mathbf{a}\right), \ldots, k\left(\mathbf{a}_{M}, \mathbf{a}\right)\right]^{T} ;$ $(\mathbf{K})_{i j}=k\left(\mathbf{a}_{i}, \mathbf{a}_{j}\right)(i, j=1, \ldots, M)$ and $k(\cdot)$ is the kernel function. A widely-used kernel function is a weighted

\footnotetext{
${ }^{3}$ Alternatively, we can use GPR to estimate the NLOS bias, but GPR is general enough to directly provide us the distance.

${ }^{4}$ It must have a zero mean and unit variance to ensure that all the features have the same influence.
}

TABLE I: The parameters estimated from the training samples.

\begin{tabular}{c||c} 
Parameter & Value \\
\hline \hline$N_{p}$ & 5000 \\
$T$ & $1 \mathrm{~s}$ \\
$\Sigma_{w}$ & $0.01 \mathbf{I}_{2} \mathrm{~m}^{2}$ \\
$\mu_{L}$ & $-0.27 \mathrm{~m}$ \\
$\sigma_{L}$ & $0.16 \mathrm{~m}$ \\
$\sigma_{N}$ & $1.61 \mathrm{~m}$ \\
$\lambda_{L}$ & $0.333 \mathrm{~ns}^{-1}$ \\
$\lambda_{N}$ & $0.075 \mathrm{~ns}^{-1}$ \\
$\left(p_{2}, p_{1}, p_{0}\right)$ & $(0.00087,-0.2,11.72)$ \\
$M$ & 450 \\
$\boldsymbol{\theta}$ & $(127.8,0.178,0.924)$ \\
$\sigma_{d}$ & $0.51 \mathrm{~m}$ \\
\hline \hline
\end{tabular}

sum of the squared-exponential and linear kernels:

$$
k\left(\mathbf{a}_{i}, \mathbf{a}_{j}\right)=\theta_{0} e^{-\theta_{1}\left\|\mathbf{a}_{i}-\mathbf{a}_{j}\right\|^{2}}+\theta_{2} \mathbf{a}_{i}^{T} \mathbf{a}_{j}
$$

where the hyperparameters $\boldsymbol{\theta}=\left(\theta_{0}, \theta_{1}, \theta_{2}\right)$, and $\sigma_{d}$, can be estimated from the NLOS training data by maximizing the log-marginal likelihood [12]. It is worth noting that, with this kernel, the correlation between the output samples is larger if the Euclidean distance between the corresponding input samples is smaller. More detailed description of GPR-based ranging is available in [10].

\section{EXPERIMENTAL RESULTS}

\section{A. Experimental Setup}

We use real measurements obtained in a basement tunnel of Linköping university. The measurement setup consisted of a vector network analyzer (VNA), two UWB omni-directional antennas, coaxial cables and a PC. We used a swept-frequency sinusoidal signal to characterize the channel between 2.5 and $4.5 \mathrm{GHz}$. The power level was set to $12 \mathrm{dBm}$. The frequency responses were transferred to the PC where a bandpass filter was used to reduce the out-of-band noise. Finally, by applying the Inverse Fast Fourier Transform, the complex impulse responses (CIR) are estimated, from which we can extract the desired features. A more detailed description of the measurement campaign is available in [6, Section III]

The deployment of the transmitters and the receivers in considered area is shown in Fig. 1. For the tracking problem, we used 6 Tx positions ( 3 LOS and 3 NLOS) representing the beacons, and $30 \mathrm{Rx}$ positions representing the moving target (we assumed a constant velocity of $1 \mathrm{~m} / \mathrm{s}$ in the horizontal direction). The NLOS condition was caused by tunnel walls built of concrete blocks with steel reinforcement. For each TxRx pair, we obtained 10 CIRs, so we obtained 1800 CIRs in total (900 LOS and 900 NLOS). Half of these samples will be used as a training data for likelihood parameters. Note that we did not perform the measurements of velocity, so we will generate the synthetic data for dynamic model. 


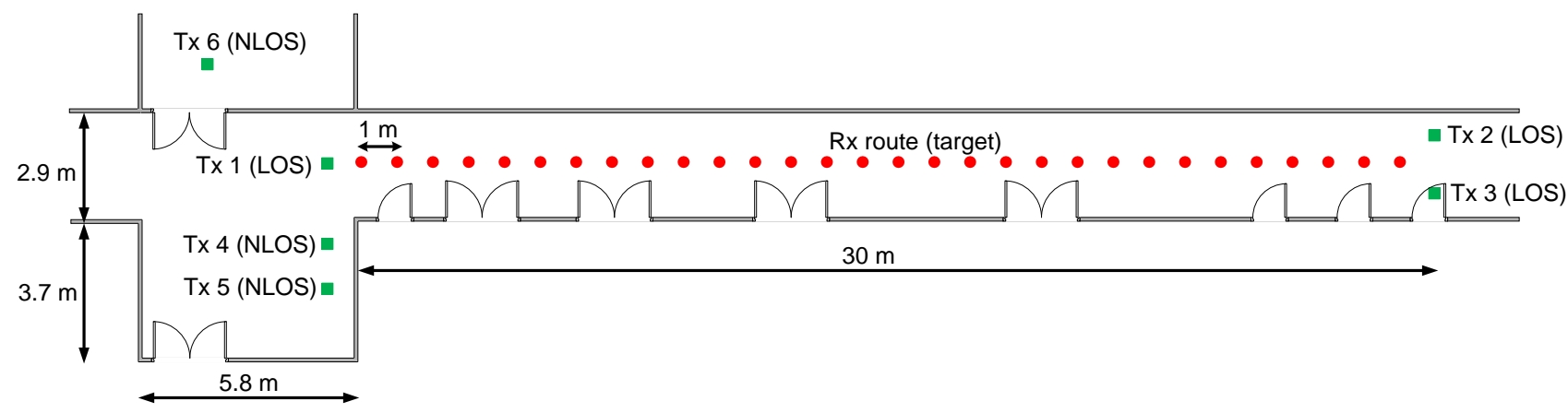

Fig. 1: Deployment of transmitters (Tx) and receivers (Rx) in the LiU tunnel. There are 6 Tx positions (3 LOS and 3 NLOS) representing the beacons, and $30 \mathrm{Rx}$ positions representing the target positions.

\section{B. Performance analysis}

We now analyze and compare the performance of the PF algorithms described in the previous sections. All required parameters are shown in Table I.

We first analyze the tracking performance with all 6 beacons (LOS+NLOS). According to Fig. 2, we can see that with TOA-based PF method the target gets lost since there is no information about NLOS bias at all (caused by 3 beacons in this case). In this case, the positioning error (up to 5 $\mathrm{m})$ is unacceptable for most applications. The TOA+Reject method provides much better results since most of the NLOS samples are rejected. However, the error is still increasing with time due to mis-classifications (there are about $20 \%$ of misclassifications according to our results from [6]). Since the error caused by mis-classifications is unknown and possibly too large, this method is not robust enough for indoor tracking. Finally, the TOA+Mitig and TOA+GPR methods provide consistently good estimates (with an error below $0.5 \mathrm{~m}$ ). The latter one is better for most percentiles 5 since it uses more features from CIR and has the ability to model complex nonlinear relationships.

Now we repeat the same analysis, but only with 3 NLOS beacons (Tx 4, Tx 5 and Tx 6). In this case, TOA+Reject is not applicable since there are no LOS measurements. The results are shown in Fig. 3. As we can see, the TOA-based method provides very poor performance since the error goes up to $8 \mathrm{~m}$. The TOA+Mitig and TOA+GPR methods again provide consistently good estimates (with an error below $1 \mathrm{~m}$ ), but now the difference between two methods is higher. This analysis shows that tracking is possible even if there is no single LOS measurement. This is a promising result, especially since many state-of-the-art indoor positioning/tracking algorithms [4] require at least 3 LOS measurements.

Finally, we provide a summary of the accuracy and complexity of all considered methods in Table II. As we can see, the complexity off all methods, except TOA+GPR, is quite similar. TOA+GPR is about 16 times slower due to the use

\footnotetext{
${ }^{5}$ In case of more general target trajectory, we expect that TOA+GPR would be the best for all percentiles.
}

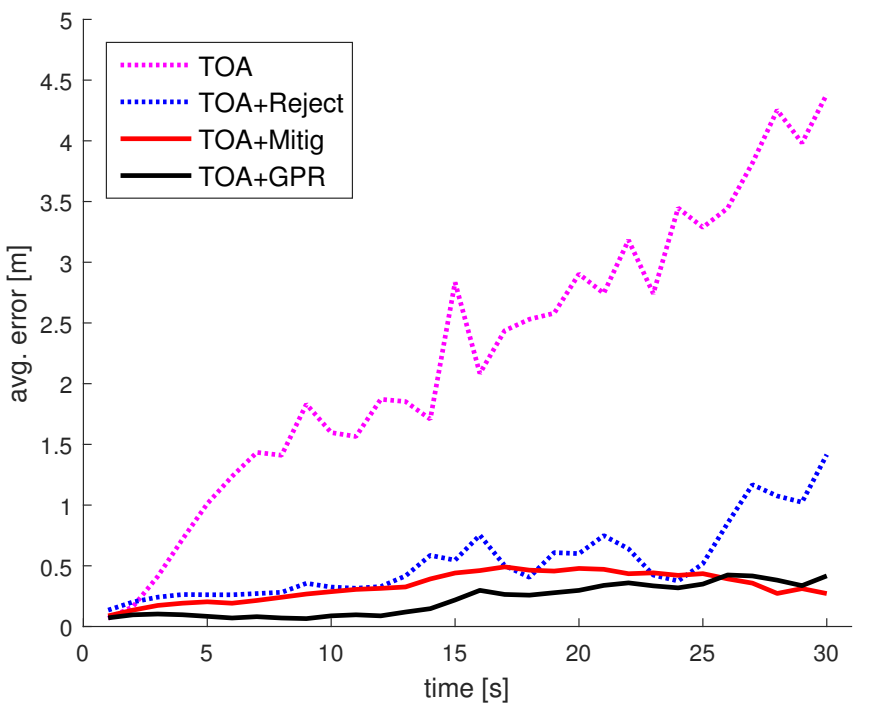

(a)

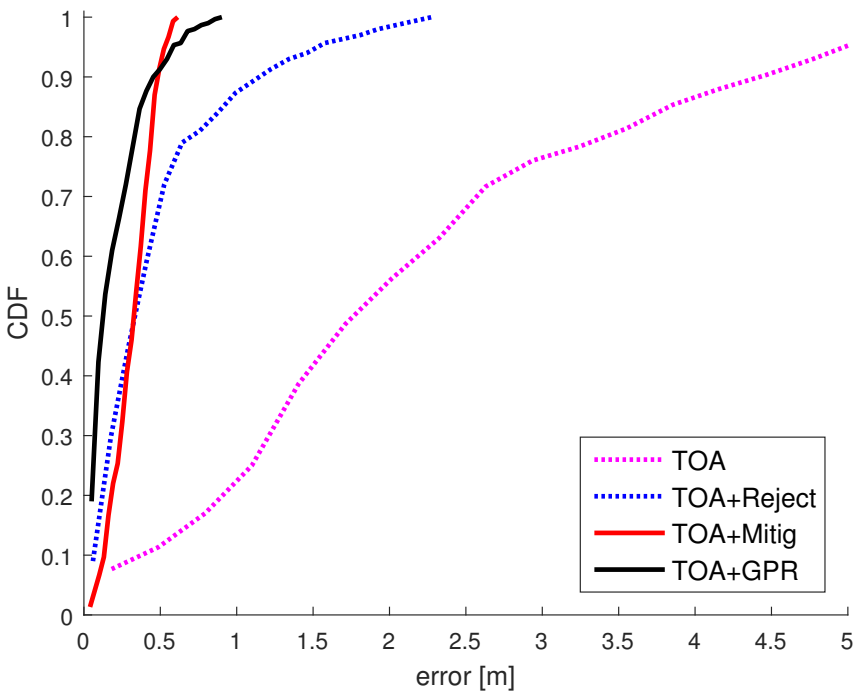

(b)

Fig. 2: Target tracking with PF with different likelihood functions. (a) Average position error as a function of time, (b) Cumulative distribution function (CDF) of the position error. 


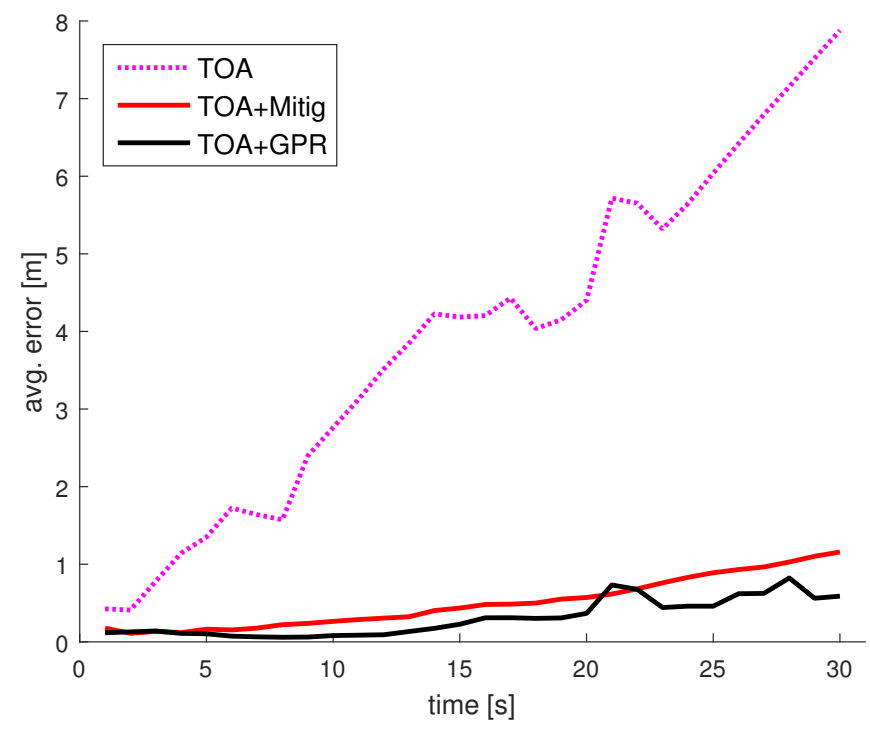

(a)

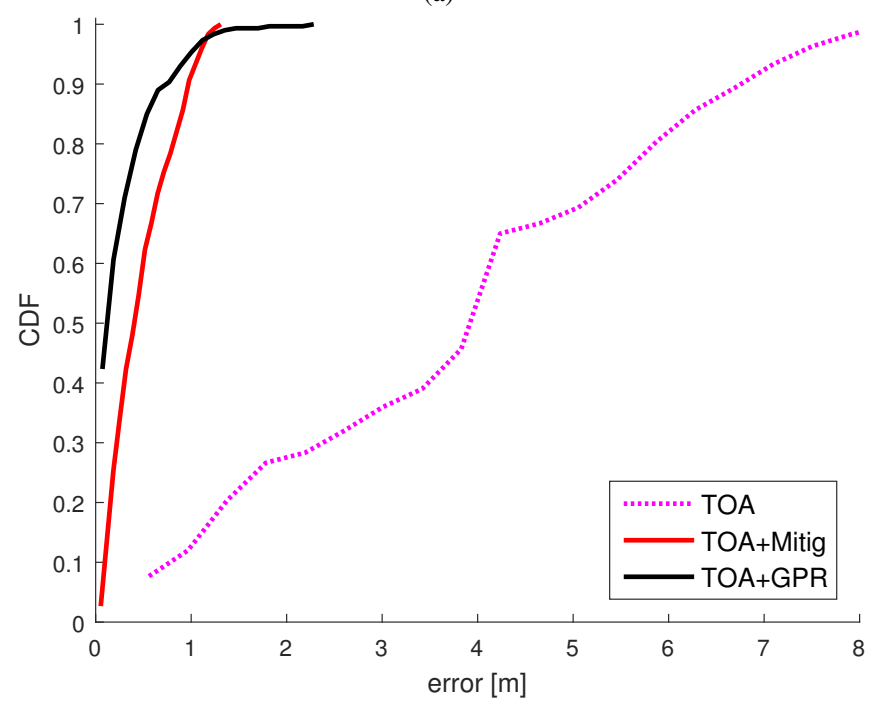

(b)

Fig. 3: Target tracking with PF with different likelihood functions and only NLOS measurements. (a) Average position error as a function of time, (b) $\mathrm{CDF}$ of the position error.

TABLE II: A comparison of accuracy and complexity of considered methods.

\begin{tabular}{c||c|c} 
Method & $\begin{array}{c}\text { accuracy }[\mathrm{m}] \\
\text { (50th/95th percentile) }\end{array}$ & complexity [ms] \\
\hline \hline TOA & $1.75 / 5.05$ & 7.2 \\
TOA+Reject & $0.32 / 1.55$ & 6.7 \\
TOA+Mitig & $0.32 / 0.53$ & 10.3 \\
TOA+GPR & $0.12 / 0.58$ & 162 \\
\hline \hline
\end{tabular}

of the training samples, but it provides the best performance. Depending on the desired trade-off between the complexity and the performance, it can be determined which of the two methods (TOA+Mitig or TOA+GPR) should be used.

\section{CONCLUSions}

We provided an experimental study of UWB indoor tracking based on PF. We considered four different likelihood functions that differ in terms of how NLOS measurements are handled. According to our results, the best performance is achieved with TOA-based ranging with GPR-based estimation for NLOS measurements, but this approach has the highest computational complexity. TOA-based ranging with mitigation of NLOS bias also provides good performance and it has a reasonably low complexity. The other two methods that we considered are not appropriate for indoor tracking due to the accumulation of error. For the future work, we will try to develop a sparse variant of the GPR-based method in order to reduce its complexity. Moreover, we will try to develop a one-step tracking method in which the range estimates are not required.

\section{ACKNOWLEDGMENT}

This work was supported by the project Cooperative Localization (CoopLoc) funded by the Swedish Foundation for Strategic Research (SSF). The authors would also like to thank Javier Ferrer-Coll (Combitech AB), Peter Stenumgaard (FOI), Per Ängskog (Univ. of Gävle) and José Chilo (Univ. of Gävle) for their help during the measurement campaign.

\section{REFERENCES}

[1] D. Dardari, P. Closas, and P. M. Djuric, "Indoor tracking: Theory, methods, and technologies," IEEE Transactions on Vehicular Technology, vol. 64, pp. 1263-1278, April 2015.

[2] L. Geng, M. F. Bugallo, A. Athalye, and P. M. Djuric, "Indoor tracking with RFID systems," IEEE Journal of Selected Topics in Signal Processing, vol. 8, pp. 96-105, Feb. 2014.

[3] S. Gezici, Z. Tian, G. B. Giannakis, H. Kobayashi, A. F. Molisch, H. V. Poor, and Z. Sahinoglu, "Localization via ultra-wideband radios: a look at positioning aspects for future sensor networks," IEEE Signal Processing Magazine, vol. 22, pp. 70-84, July 2005.

[4] I. Guvenc and C.-C. Chong, "A survey on TOA based wireless localization and NLOS mitigation techniques," IEEE Communications Surveys \& Tutorials, vol. 11, no. 3, pp. 107-124, 2009.

[5] J. Khodjaev, Y. Park, and A. S. Malik, "Survey of NLOS identification and error mitigation problems in UWB-based positioning algorithms for dense environments," Annals of telecommunications, vol. 65, no. 5-6, pp. 301-311, 2010.

[6] V. Savic, J. Ferrer-Coll, P. Angskog, J. Chilo, P. Stenumgaard, and E. G. Larsson, "Measurement analysis and channel modeling for TOA-based ranging in tunnels," IEEE Trans. on Wireless Communications, vol. 14, pp. 456-467, Jan. 2015.

[7] V. Savic, H. Wymeersch, and S. Zazo, "Belief consensus algorithms for fast distributed target tracking in wireless sensor networks," Signal Processing, vol. 95, pp. 149-160, Feb. 2014.

[8] G. Welch and G. Bishop, "An introduction to the Kalman filter," tech. rep., University of North Carolina at Chapel Hill, July 2006.

[9] M. S. Arulampalam, S. Maskell, N. G. Gordon, and T. Clapp, "A tutorial on particle filters for online nonlinear/non-Gaussian Bayesian tracking,' IEEE Transactions on Signal Processing, vol. 50, pp. 174-188, Feb. 2002.

[10] V. Savic, E. G. Larsson, J. Ferrer-Coll, and P. Stenumgaard, "Kernel methods for accurate UWB-based ranging with reduced complexity," IEEE Transactions on Wireless Communications, vol. 15, pp. 17831793, March 2016.

[11] L. Yi, S. G. Razul, Z. Lin, and C. M. See, "Target tracking in mixed los/nlos environments based on individual measurement estimation and los detection," IEEE Transactions on Wireless Communications, vol. 13, pp. 99-111, Jan. 2014.

[12] C. E. Rasmussen and C. K. I. Williams, Gaussian Processes for Machine Learning. MIT Press, 2006. 